\title{
Cytotoxic Effect of Cord Blood Derived Natural Killer Cells on Breast Cancer Cells
}

\author{
(D) Ayfer KARLITEPE, ${ }^{1}$ (D) Tolga ATAKUL, ${ }^{2}$ (i) Mehtap KILIÇ EREN' \\ 'Department of Medical Biology, Aydın Adnan Menderes University Faculty of Medicine, Aydın-Turkey \\ ${ }^{2}$ Department of Gynecology and Obstetrics, Aydın Adnan Menderes University Faculty of Medicine, Aydın-Turkey
}

\begin{abstract}
OBJECTIVE
Human natural killer (NK) cells are cluster of differentiation 3 (CD3)-CD56 + lymphocytes. NK cells can be obtained from various sources such as peripheral blood and cord blood. Among those, cord blood (CB) is an important cellular resource. The aim of this study is to determine the cytotoxic effect of freshly isolated CB derived NK cells toward breast cancer cells in vitro.
\end{abstract}

\section{METHODS}

CB mononuclear cells were isolated by Ficoll-Paque and NK cells were selected by with magnetic activated cell sorting (MACS) technique. CB-NK cell's surface markers were quantified by flow cytometry analysis. Water-soluble tetrazolium salt cytotoxicity assay was used to measure cell viability; Annexin V/7-amino actinomycin D assay was used to measure apoptosis and necrosis. Enzyme-linked immunosorbent assay was used to determine perforin and Granzyme B activity of CB-NK cells.

\section{RESULTS}

Here, we show that CD56+ cells within the NK cell population were measured as $99.59 \%$ and CD314+ cells surface marker expression was measured as $99.48 \%$ after MACS selection. CB-NK cells exerted significant cytotoxicity toward Michigan cancer foundation 7 (MCF-7), MDA-231, and K562 tumor cells. Importantly, we show that CB-NK cells were able to kill MCF-7 and MDA-231 cells through apoptosis and necrosis, respectively. The amount of Granzyme B and perforin produced by CB-NK cells was measured as $50 \mathrm{ng} / \mathrm{ml}$ and $80 \mathrm{ng} / \mathrm{ml}$, respectively.

\section{CONCLUSION}

Our findings confirm that freshly isolated CB-NK cells can be expanded in vitro with supplementation of various cytokines. We provide evidence that in these conditions, CB-NK cells exert efficiently cytotoxic effect and induced apoptotic and necrotic cell death toward breast cancer cells.

Keywords: Breast cancer; cancer immunotherapy; fresh cord blood natural killer cells.

Copyright $\odot$ 2021, Turkish Society for Radiation Oncology

\section{Introduction}

Natural killer (NK) cells are produced in the bone marrow and settled in various lymphoid and non-lymphoid tissues including lymph nodes, spleen, peripheral blood, lung, and liver.[1] Human NK cells are cluster of differentiation 3 (CD3)-CD56 + lymphocytes. [2]
Having many cytotoxic receptors that are effective in recognizing malignant cells. These receptors are called NKp46, NKp44, NKp30, NKG2A/B/C/CD94, NKG2D/ CD314, KIR-2DS, KIR3DS, KIR-2DL, and KIR-3DL. [3] NK cells are activated by cytokines such as interleukin 2 (IL 2), IL 12, IL 15, and IL 18 and can induce apoptosis in tumor cells by several mechanisms. The 
most common tumor cell death mechanism is the induction of apoptosis in cancer cells through cytotoxic proteins secreted by NK cells such as perforin and granzyme $\mathrm{B}$ and cell death receptor and ligand interaction (Fas/FasL, etc.).[4] NK cells can be obtained from various sources including peripheral blood cord blood (CB) and bone marrow hematopoietic stem cells and can be differentiated from embryonic and induced pluripotent stem cells (IPS) which are important in cellular therapy today. [5]

$\mathrm{CB}$ is an important cellular resource of allogenic NK cells and can be preserved for many years with the cryopreservation technique.[6] CB has important advantages such as being suitable for allogenic use and being easily obtained.[7] CB derived NK cells displays the same quality as peripheral blood derived NK cells in terms of the amount of interferon gamma (INF $\gamma$ ) and tumor necrosis factor alpha production as well as expression of cytotoxic receptors. [8,9] In addition, $\mathrm{CB}$ and peripheral blood derived NK cells can be induced by same IL2, IL7, IL15, IL18, and IL21 for in vitro expansion.[10]

Breast cancer is one of the leading cancer deaths among women;[11] thus, alternative treatment approaches are in development in addition to chemotherapy and radiotherapy.[12] Recently, immunotherapy approach in breast cancer treatment has attracted considerable attention.

Here, in this study, we isolated NK cells from umbilical CB and expanded them in vitro by cytokine supplementation and eventually assessed the cytotoxic ability of CB-derived NK cells on breast cancer cells in vitro.

\section{Materials and Methods}

\section{Ethics Statement}

$\mathrm{CB}$ samples were obtained from cesarean deliveries after informed consent. The study complied with all provisions of the Declaration of Helsinki and were approved by non-interventional clinical research ethics committee of Aydin Adnan Menderes University Medicine Faculty, Aydın, Turkey (Ethics Committee Apr. No. 2018/1354).

\section{Cell Culture}

Michigan Cancer Foundation-7 (MCF-7) (ATCC ${ }^{\circledast}$ HTB-22 $^{\mathrm{mm}}$ ), MDA-231 (ATCC ${ }^{\circledR} \mathrm{HTB}^{-26^{\mathrm{mw}}}$ ) breast cancer cells, and the leukemia cell line K562 (ATCC ${ }^{\circledR}$ CCL$243^{\text {mix }}$ ) were obtained from American Type Culture Collection cultured in Roswell Park Memorial Institute (RPMI) Medium (Gibco, USA) supplemented with $10 \%$ fetal bovine serum (Gibco, USA) and $1 \%$ penicillin/streptomycin (Gibco, USA).

\section{Mononuclear Cell (MNC) Isolation and Culture}

Six freshly collected CB (50 ml/unit) were layered over Ficoll Histopaque (Sigma, USA) and centrifuged at $400 \mathrm{~g}$ for $30 \mathrm{~min}$. MNCs were collected at the interface and washed in phosphate-buffered saline (PBS) (Gibco, USA). CB MNCs were cultured in RPMI-1640 medium supplemented $500 \mathrm{IU} / \mathrm{mL}$ IL2 (ProSpec, Israel) for 1 week.[13]

\section{Isolation and Expansion of NK Cells}

CD56 positive NK cells from CB MNCs were isolated by immunomagnetic bead selection using the magnetic activated cell sorting (MACS) system according to the manufacturer's instructions. CD56+ CB-NK cells were cultured in RPMI-1640 medium supplemented $500 \mathrm{IU} / \mathrm{mL}$ IL2, 50 $\mathrm{ng} / \mathrm{ml}$ stem cell factor (SCF) (ProSpec, Israel), $50 \mathrm{ng} / \mathrm{ml}$ fms-like tyrosine kinase 3 (FLT3) (ProSpec, Israel), and $40 \mathrm{ng} / \mathrm{ml}$ IL15 (ProSpec, Israel) cytokines for 1 week.[14]

\section{Flow Cytometry Analysis}

$3 \times 104$ CB-NK cells were washed with PBS and incubated with phycoerythrin (PE) conjugated anti-CD56 antibody (1:10 dilution; BioLegend, USA), PE conjugated anti-CD314 antibody (1:10 dilution; BioLegend, USA), and isotype control antibody PE conjugated IgG1 antibody (1:10 dilution; BioLegend, USA) for $20 \mathrm{~min}$ at $4^{\circ} \mathrm{C}$. Then, the cells were washed with PBS and surface markers expressions were analyzed by flow cytometry (Beckman Coulter, Life Sciences, USA).

\section{Cytotoxicity Assay Water-soluble Tetrazolium Salt (WST1)}

CB-NK, MCF-7, MDA-231, and K562 control cells or CB-NK cells mixed with tumor cells (MCF-7, MDA-231, and K562) as 1:1 and 1:2 (effector:target/E:T) ratios were plated at $10^{4}$ cells/per well and in 96 well plates in a final volume of $100 \mu \mathrm{l} /$ well culture medium in a humidified atmosphere $\left(37^{\circ} \mathrm{C}\right.$ and $\left.5 \% \mathrm{CO}_{2}\right)$. Cells were incubated for $4 \mathrm{~h}$ and following $10 \mu \mathrm{l}$ Cell Proliferation Reagent WST1 (Sigma, USA) was added to each well and incubated for $30 \mathrm{~min}$ at $37^{\circ} \mathrm{C}$ and $5 \% \mathrm{CO}_{2}$. The wavelength to measure absorbance was $450 \mathrm{~nm}$ on the microplate reader. The percentage of cytotoxicity was calculated from the following equation using corrected absorbance: \% Cytotoxicity $=(\times 100$ (Control-Sample) $) /$ Control.

\section{Apoptosis Analysis (Annexin V/7-Amino Actino- mycin D [7AAD] Assay)}

CB-NK cells were mixed with tumor cells (MCF-7, MDA-231, and K562) at 1:1 and 1:2 ratios. MCF-7, MDA-231, and K562 tumor cells were plated at 105 

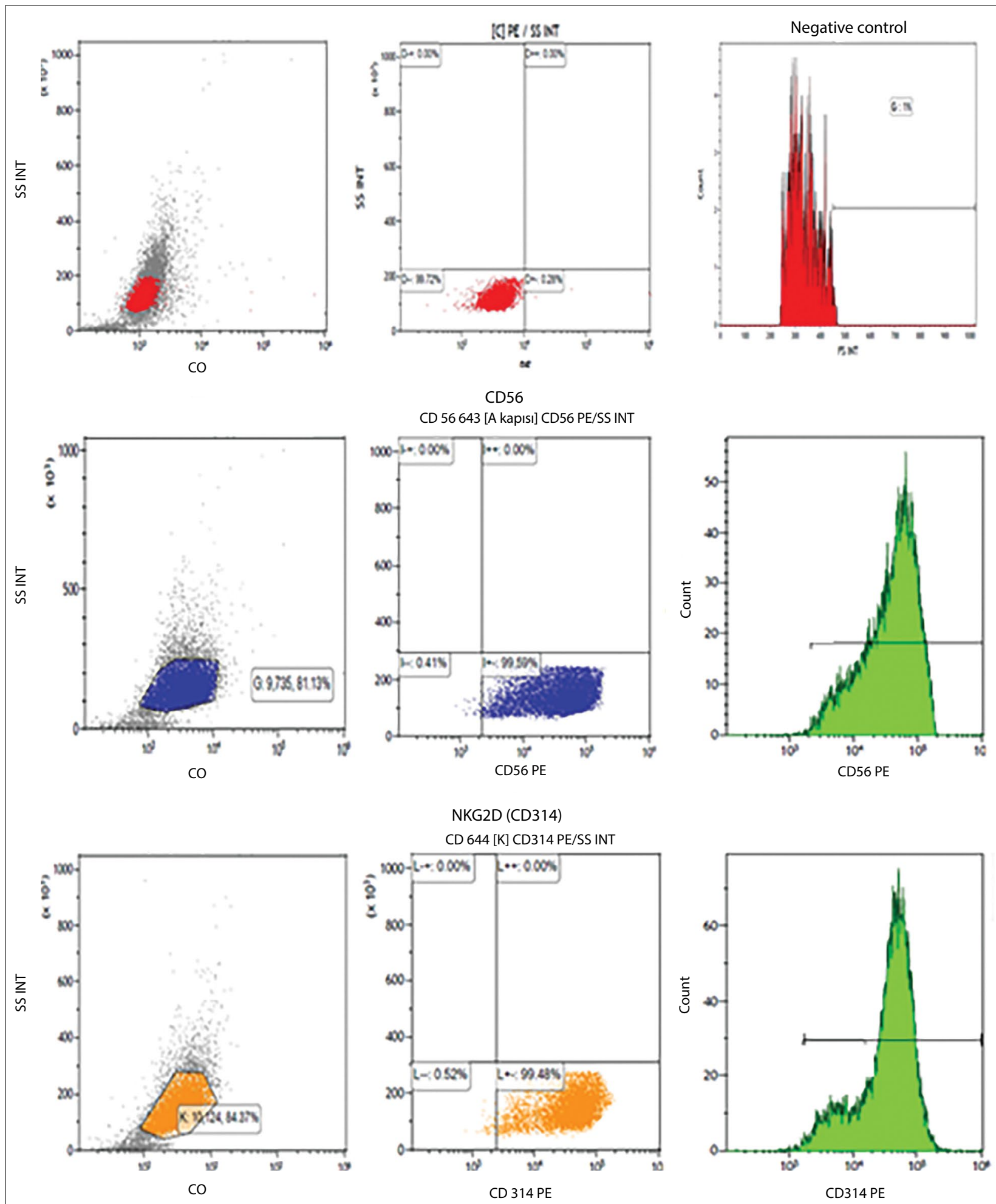

Fig. 1. The surface markers expression on CB-MNC and CB-NK cells. Flow cytometry was used to assess surface expression of CD56 and NKG2D after magnetic activated cell sorting (MACS) separation. Negative Control, CB-NK cell CD56 exp. after MACS, CB-NK cell CD314 exp. after MACS.

CO: Control (Ig G), PE: Phycoerythrin. 


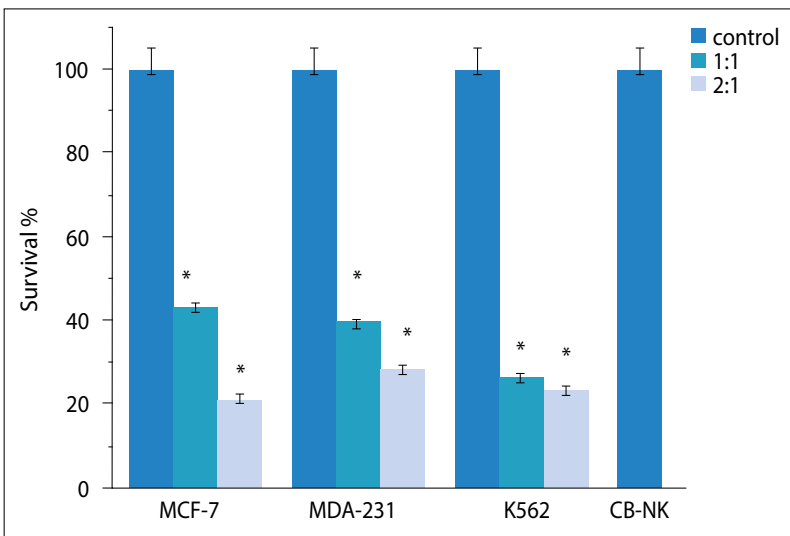

Fig. 2. Cytotoxic activity of CB-NK cells. Michigan cancer foundation 7, MDA-231, and K562 cells were incubated with CB-NK cells for $4 \mathrm{~h}$ at 1:1 and 1:2 Effector: Target ratios. WST-1assay used for measurement of cell viability. $\left({ }^{*} \mathrm{p}<0.0001\right.$, Target vs. Targer+CB-NK).

MCF: Michigan cancer foundation, MDA: MD Anderson, CB-NK: Cord blood derived natural killer cells.

cells/per well and then CB-NK cells were added and mixed in 46 well plates and cells were incubated for $4 \mathrm{~h}$ at $37^{\circ} \mathrm{C}$ in a humidified atmosphere in a $\mathrm{CO}_{2}$ incubator. Determination of apoptosis profile was achieved using Muse ${ }^{\oplus}$ Annexin V and Dead Cell Assay according to the manufacturer's instructions. Quantitative analysis of apoptotic and necrotic cells was evaluated by Muse Cell Analyzer (Millipore, Austin, TX, USA).

\section{Measurement of Cytokine Production by Enzyme- linked Immunoabsorbent Assay (ELISA)}

CB-NK cells were plated at $10^{5}$ cells/per well in 46 well plates and cells were incubated for $24 \mathrm{~h}$ at $37^{\circ} \mathrm{C}$ in humidified atmosphere in a $\mathrm{CO}_{2}$ incubator. $\mathrm{Su}$ pernatants were collected from CB-NK cells after incubation and Granzyme B and perforin levels were measured using commercial ELISA kits (Booster, China), according to the manufacturer's instructions. Granzyme B and perforin levels in the supernatant were determined by measuring absorbance at $450 \mathrm{~nm}$ on a microplate reader.

\section{Statistical Analysis}

Statistical analysis was performed using Origin 8, Graph software. Paired sample student's t-test was used where indicated $\left({ }^{*} \mathrm{p}<0.001 ;{ }^{*} \mathrm{p}<0.0001\right)$. Data are expressed as means of three independent experiments $\pm \mathrm{SD}$ (standard deviation).

\section{Results}

\section{CB-NK Cells Characterization}

To examine the efficacy of CB-NK cells on breast cancer cells in vitro; initially, we isolated that NK cells characterization of CB-NK cells was performed with flow cytometry after immunomagnetic bead MACS selection. CD56+ cells were measured as $99.59 \%$ within NK cell population after MACS selection (Fig. 1). In addition, CD314+ cells surface marker expression was measured as $99.48 \%$ after MACS selection (Fig. 1).

\section{CB-NK Cells Exert Cytotoxicity Towards Breast Cancer Cell Lines}

We tested the cytotoxicity of in vitro expanded CB-NK cells toward MCF-7, MDA-231 cells using WST1 cytotoxicity assay. K562 cells were used as positive control which is known from literature.[15] As shown in Figure 2, our results demonstrate that $\mathrm{CB}-\mathrm{NK}$ cells were able to lyse MCF-7, MDA-231 cell. CB-NK cells show variable cytotoxicity toward MCF-7, MDA-231, and K562 tumor cells. CB-NK cells lyse MCF- 7 cells by $57 \%$ and MDA-231 cells by $60 \%$. It was also $74 \%$ effective against K562 leukemia cells (Fig. 2).

\section{CB-NK Cells Induced Apoptosis and Necrosis in Breast Cancer Cell Lines}

To determine whether CB-NK cells induced cytotoxicity is mediated by cell death, we used Annexin V/7AAD assay. As shown in Figure 3, our results demonstrate that CB-NK cells were able to kill MCF-7 cell, by apoptotic cell death and apoptosis were detected as $50 \%$. Interestingly, in MDA-231 cells, we detected mainly necrotic cell death. CB-NK induced necrotic cell death rate was 35\% in MDA 231 breast cancer cells. In addition, when we measure the cell death in K562 cell line, we detected $63 \%$ of apoptosis. Thus, these results confirmed that CB-NK cells were able to kill breast cancer cell lines effectively (Fig. 3).

\section{CB-NK Cells Produced Cytotoxic Proteins}

Since we measured considerable amount of cell death against breast cancer cell lines, we measured whether CB-NK cell produced cytotoxic proteins Granzyme B and perforin. We measured the amount of Granzyme $B$ and perforin by ELISA. Our results show that CBNK cells produce different levels of Granzyme $B$ and perforin. As shown in Figure 4, CB-NK cells produced $50 \mathrm{ng} / \mathrm{ml} \mathrm{Granzyme} \mathrm{B} \mathrm{and} 80 \mathrm{ng} / \mathrm{ml}$ perforin cytotoxic proteins (Fig. 4). 


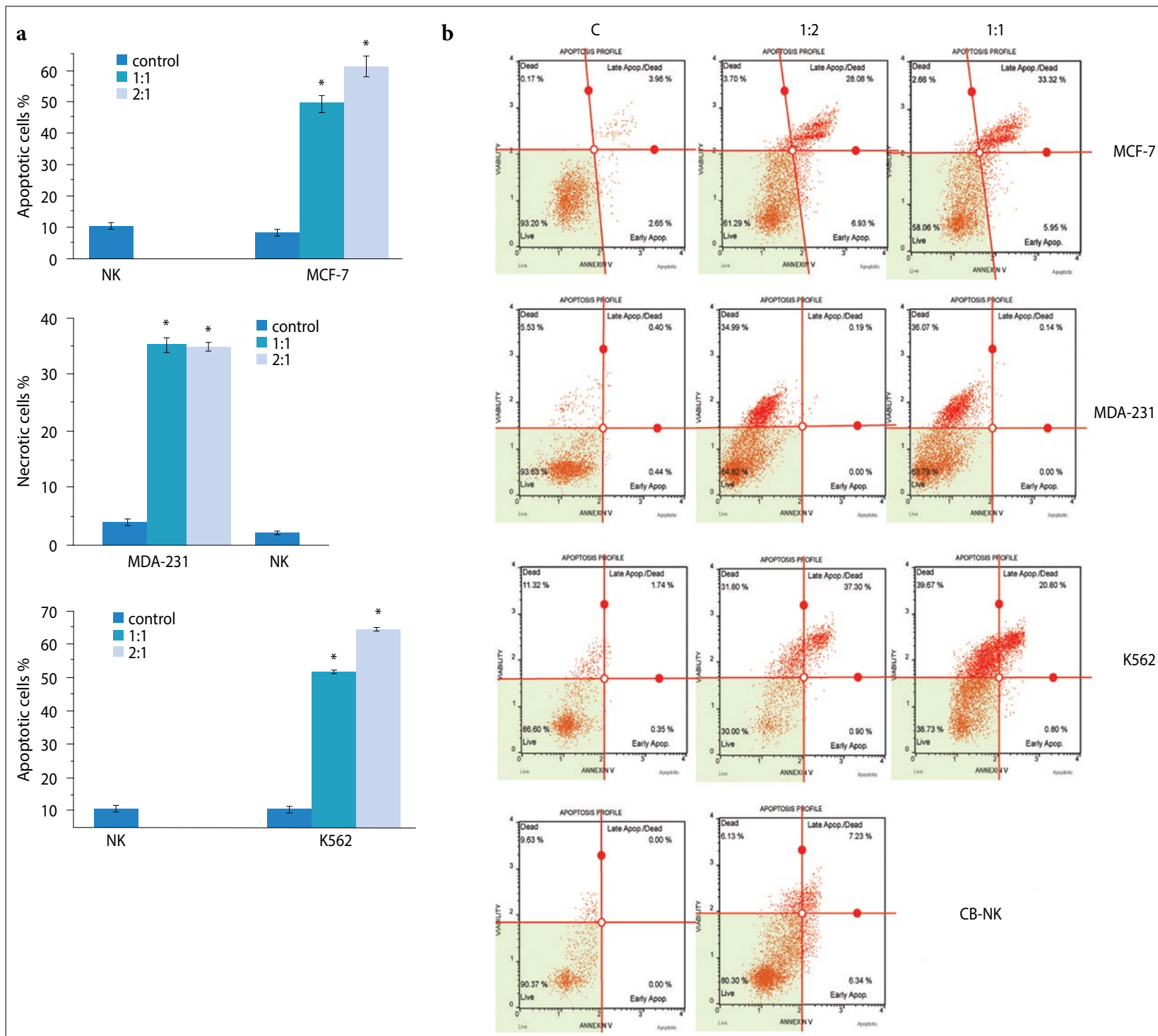

Fig. 3. (a) Quantification of apoptotic cells. (b) Flow cytometry plots. CB-NK cells induce apoptosis Michigan Cancer Foundation 7 (MCF-7) and K562 cells while induce necrosis MDA-231 cells. CB-NK cells and tumor target cells were incubated for $4 \mathrm{~h}$ at 1:1 and 2:1 E:T ratios and used Muse Annexin V and Dead Cell Assay. Apoptotic cell death MCF-7, Necrotic cell death MDA-231, Apoptotic cell death K562, and Control CB-NK cell $\left({ }^{*} \mathrm{p}<0.0001\right)$. MCF: Michigan cancer foundation, MDA: MD Anderson, CB-NK: Cord blood derived natural killer cell, NK: Natural killer.

\section{Discussion}

NK cells are important mediators of immunotherapy approach and can be obtained from several sources including peripheral blood, $\mathrm{CB}$ and bone marrow hematopoietic stem cells, and embryonic or IPS.[5] Among those $\mathrm{CB}$ contains considerable amount of NK cells and has been considered as a promising source of NK cells for cellular immunotherapy. CB offers unique advantages, which are directly applicable to NK cell-directed allore- activity. The ease of collection of CB and cryopreservation possibilities makes them attractive source for NK cell immunotherapy. However, it has not been easy to expand a large number of high purity NK cells from CB; thus, various methods have been developed.[7-9]

Breast cancer is the most common type of cancer in women.[15] At present, cellular immunotherapy approach has gained great importance as significant therapeutic option in many other cancers including breast cancer. Although significant advances have 


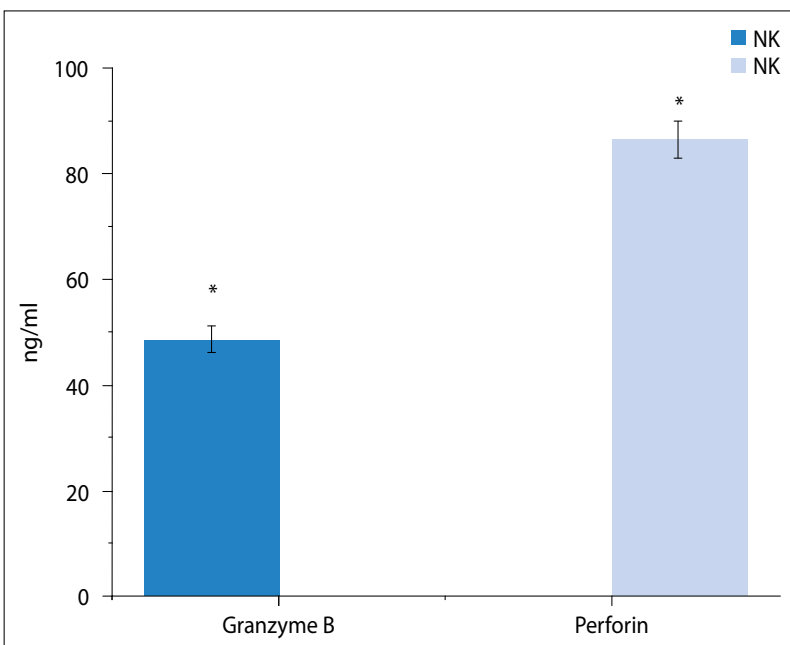

Fig. 4. CB-NK cell produced cytotoxic granules Granzyme B and perforin. Supernatants were collected from CB-NK cells after incubation, Granzyme B and perforin levels using the enzyme-linked immunosorbent assay kits. Granzyme B and perforin enzyme-linked immunoabsorbent assay $\left({ }^{\star} \mathrm{p}<0.001\right)$. NK: Natural killer.

been made in immunotherapy, more studies are still needed in breast cancer. We are interested in using human CB derived NK cells as an alternative source of immunotherapy against human breast cancers. Here, we show that freshly isolated and in vitro expanded CB-NK cells can efficiently kill MCF-7 and MDA-231 breast cancer cells in vitro.

NK cells found in peripheral blood at rate of $10 \%$ whereas in $\mathrm{CB}$ it is $30 \%$.[8] NK cells are characterized by their CD3-CD56+ surface marker expressions.[1] In our study, we isolated MNCs from $\mathrm{CB}$ according to CD56 positivity by magnetic separation and show that we can obtain a pure NK cell population by this technique (Fig. 1). Inhibitory and activator receptor expressions are of great importance in the regulation of NK cell functions. Peripheral blood and CB NK cells show a similar rate of NKG2D activator receptor (also known as CD314) expression.[16] Our result shows that NKG2D activator receptor is the high level of expression pattern (Fig. 1).

Many culture protocols have been developed for the growth of CB NK cells in vitro. Among these protocols; using different cytokine combinations SCF, FLT3 ligand, IL2, IL7, IL12, IL15, and IL18 and special feeder layers (CD3dep PBMC, antigen-presenting cell, mbIL$21 \mathrm{~K} 562$, etc.) are more common.[17-20] In our study, we used cytokine cocktail including SCF, FLT3 ligand, IL2, and IL15 for CB-NK cells culture.
In a previous study, Nham et al. have shown that CB NK cells have different cytotoxic effects on triple negative breast cancers and hormone positive breast cancer cells [14]. The presence of factors such as human leukocyte antigen-I (HLAI) and HLAG antigens and immunosuppressive factor transforming growth factor-beta expression or inhibitory receptor NKG2A expression, has been shown as limiting factors of the cytotoxic effect of NK cells. [14] In line with the previous studies, we also show that CB-NK cells have different levels of cytotoxic effects on breast cancer cells (Fig. 2). However, whether or not the same factors might be responsible for the differences will be future subject of our studies. One of the important finding of our study is that CB-NK cells induce apoptosis in MCF-7 cell (Fig. 3) but necrosis in MDA231 cells (Fig. 3). Recently, Backes et al. show that NK cells are capable of inducing apoptosis, necrosis, and other mix forms of cell death in tumor cells $[21,22]$. Furthermore, it was found that mainly two major cytotoxic pathways perforin/granzyme release or FasL/FasR interaction mediates the cell death. On the other hand, it was shown that these pathways may suppress each other's effect depending on the amount of calcium entering to the cell.[21] In our case, the reason why CB-NK cells induced different types of cell death in different cell lines may be dependent on cell type or context. At present, we do not know the exact mechanism; further studies are needed to clarify these issues.

Our results demonstrating CB-NK cells produced perforin and Granzyme B cytotoxic proteins suggest that CB-NK mediated cytotoxic activity may be related to granzyme and perforin activity (Fig. 4). Previously, it was shown that NK cells exert cytotoxic activity by Granzyme B and perforin pathway.[1]

\section{Limitations of the Study}

In this study, the cytotoxic activity of CB derived NK cells on breast cancer cells was determined. However, by which mechanisms CB-NK cells induce apoptosis (INF $\gamma$ or death cell receptor/ligand interactions) has not been evaluated in tumor cells.

\section{Conclusion}

$\mathrm{CB}$ is an important resource for cancer immunotherapy approaches because of its NK cell content. In our study, we investigated the effect of fresh CB-NK cells with common methods. Our findings confirm that freshly isolated CB-NK cells can be in vitro expanded 
solely with various cytokine combinations including SCF, Flt3 IL2, and IL15. In addition, we provide evidence that CB-NK cells exert efficiently cytotoxic effect and induced apoptotic and necrotic cell death toward breast cancer cells. Our study suggest that fresh $\mathrm{CB}$ can be used as an alternative resource for NK cell isolation which can be further expanded and used for cancer immunotherapy approaches. Future studies are needed to determine exact mechanism of cytotoxic activity of CB-NK cells.

Acknowledgments: We are thankful to Adnan Menderes University Scientific Research Foundation (BAP) for supporting the study (TPF-18043). We also thank to Ercüment Dirice (Adnan Menderes University Hospital, Dept. of Hematology) for his technical help on Flow Cytometry Analysis.

Peer-review: Externally peer-reviewed.

Conflict of Interest: The authors declare that they have no conflict of interest.

Ethics Committee Approval: The study was approved by the Non-interventional Clinical Research Ethics Committee of Aydin Adnan Menderes University Faculty of Medicine (No: 24, Date:12/04/2018).

Financial Support: This study was supported by Aydin Adnan Menderes University Scientific Research Foundation (BAP) TPF18043.

Authorship contributions: Concept - M.K.E., A.K.; Design - M.K.E., A.K.; Supervision - M.K.E.; Funding M.K.E.; Materials - M.K.E., A.K., T.A.; Data collection and/ or processing - M.K.E., A.K.; Data analysis and/or interpretation - M.K.E., A.K., T.A.; Literature search - M.K.E., A.K., T.A.; Writing - M.K.E.; Critical review - M.K.E., A.K., T.A.

\section{References}

1. Cooper MA, Fehniger TA, Caligiuri MA. The biology of human natural killer-cell subsets. Trends Immunol 2001;22(11):633-40.

2. Cheng M, Chen Y, Xiao W, Sun R, Tian Z. NK cell-based immunotherapy for malignant diseases. Cell Mol Immunol 2013;10(3):230-52.

3. Anfossi N, Andre P, Guia S, Falk CS, Roetynck S, Stewart $\mathrm{CA}$, et al. Human NK cell education by inhibitory receptors for MHC class I. Immunity 2006;25(2):331-42.

4. Smyth MJ, Cretney E, Kershaw MH, Hayakawa Y. Cytokines in cancer immunity and immunotherapy. Immunol Rev 2004;202:275-93.

5. Grossenbacher SK, Aguilar EG, Murphy WJ. Leveraging natural killer cells for cancer immunotherapy. Immunotherapy 2017;9(6):487-97.
6. Sarvaria A, Jawdat D, Madrigal JA, Saudemont A. Umbilical cord blood natural killer cells, their characteristics, and potential clinical applications. Front Immunol 2017;23(8):329.

7. Mehta R, Shpall E, Rezvani K. Cord blood as a source of natural killer cells. Front Med (Lausanne) 2016;2:93.

8. Luevano M, Daryouzeh M, Alnabhan R, Querol S, Khakoo S, Madrigal A, et al. The unique profile of cord blood natural killer cells balances incomplete maturation and effective killing function upon activation. Hum Immunol 2012;73(3):248-57.

9. Verneris MR, Miller JS. The phenotypic and functional characteristics of umbilical cord blood and peripheral blood natural killer cells. Br J Haematol 2009;147(2):185-91.

10. Perez SA, Sotiropoulou PA, Gkika DG, Mahaira LG, Niarchos DK, Gritzapis AD, et al. A novel myeloidlike nk cell progenitor in human umbilical cord blood. Blood 2003;101(9):3444-50.

11. Garnock-Jones KP, Keating GM, Scott LJ. Trastuzumab: a review of its use as adjuvant treatment in human epidermal growth factor receptor 2 (HER2)positive early breast cancer. Drugs 2010;70(2):215-39.

12. Yao H, He G, Yan S, Chen C, Song L, Rosol T, et al. Triple-negative breast cancer: is there a treatment on the horizon? Oncotarget 2017;8(1):1913-24.

13. Alnabhan R, Madrigal A, Saudemont A. Differential activation of cord blood and peripheral blood natural killer cells by cytokines. Cytotherapy 2015;17(1):7385.

14. Nham T, Poznanski MS, Fan I, Vahedi F, Shenouda $\mathrm{M}$, Lee A, et al. Ex vivo-expanded natural killer cells derived from longterm cryopreserved cord blood are cytotoxic against primary breast cancer cells. J Immunother 2018;41(2):64-72.

15. Li Y, Schmidt-Wolf I, Wu Y, Huang S, Wei J, Fang J, et al. Optimized protocols for generation of cord blood-derived cytokine-induced killer/natural killer cells. Anticancer Res 2010;30(9):3493-9.

16. Wang $\mathrm{Y}$, Xu H, Zheng X, Wei H, Sun R, Tian Z. High expression of NKG2A/CD94 and low expression of granzyme $\mathrm{b}$ are associated with reduced cord blood NK cell activity. Cell Mol Immunol 2007;4(5):377-82.

17. Granzin M, Wagner J, Köhl U, Cerwenka A, Huppert V, Ullrich E. Shaping of natural killer cell antitumor activity by ex vivo cultivation. Front Immunol 2017;8:458.

18. Koehl U, Brehm C, Huenecke S, Zimmermann SY, Kloess S, Bremm M, et al. Clinical grade purification and expansion of nk cell products for an optimized manufacturing protocol. Front Oncol 2013;3:118.

19. Denman CJ, Senyukov VV, Somanchi SS, Phatarpekar PV, Kopp LM, Johnson JL, et al. Membrane-bound IL21 promotes sustained ex vivo proliferation of human 
natural killer cells. PLoS One 2012;7(1):e30264.

20. Ahn YO, Kim S, Kim TM, Song EY, Park MH, Heo DS. Irradiated and activated autologous PBMCs induce expansion of highly cytotoxic human NK cells in vitro. J Immunother 2013;36(7):373-81.

21. Backes C, Friedmann K, Mang S, Knörck A, Hoth M, Kummerow C. Natural killer cells induce distinct modes of cancer cell death: discrimination, quantification, and modulation of apoptosis, necrosis, and mixed forms. J Biol Chem 2018;293(42):16348-63.

22. Joshi S, Tarantolo S, Kuszynski C, Kessinger A. Antitumor therapeutic potential of activated human umbilical cord blood cells against leukemia and breast cancer. Clin Cancer Res 2000;6(11):4351-8. 